\title{
Sobre el mal de los ardientes o del fuego de San Antonio
}

\author{
Saint Anthony's fire illness
}

Juan David Ramírez-Quintero • Medellín (Colombia)

\section{Resumen}

El ergotismo es el resultado de la intoxicación con alcaloides derivados del ergot. Durante la edad media y hasta el siglo XIX fue el responsable de grandes epidemias en Europa por el consumo de granos contaminados con el cornezuelo del centeno provocando manifestaciones isquémicas características y afección del sistema nervioso. El ergotismo como se conoció en el Medioevo ha desaparecido, pero aún hoy en día se reportan casos en el marco de uso terapéutico de ergotamínicos. Se pretenden describir algunos de sus aspectos históricos y clínicos distintivos. (Acta Med Colomb 2018; 43: 156-160).

Palabras clave: ergotismo, claviceps, gangrena, isquemia, convulsiones

\begin{abstract}
Ergotism is the result of intoxication with alkaloids derived from ergot. During the middle ages and until the nineteenth century it was responsible for major epidemics in Europe for the consumption of grains contaminated with ergot, causing characteristic ischemic manifestations and nervous system involvement. Ergotism as it was known in the Middle Ages has disappeared, but even today cases are reported in the therapeutic use of ergotamine. Some of its distinctive historical and clinical aspects are intended to be described. (Acta Med Colomb 2018; 43: 156-160).

Key words: ergotism, claviceps, gangrene, ischemia, seizures
\end{abstract}

Dr. Juan David Ramírez-Quintero: Médico Internista. Universidad de Antioquia. Hospital Pablo Tobón Uribe. Seguros Sura. Medellín. Correspondencia. Dr. Juan David RamírezQuintero, Medellín (Colombia).

Correo electrónico: jdramirezq@yahoo.com Recibido: 23/I/2017 Aceptado: 25/VI/2018

\section{Introducción}

Cuando la práctica médica deja de ser un encuentro dialógico y comprensivo pierde su núcleo fundacional, materializándose las palabras de Montaigne en su ensayo "De la presunción": "No somos más que ceremonia, nos arrastra la ceremonia y olvidamos la sustancia de las cosas; nos quedamos con las ramas y abandonamos el tronco y el cuerpo" (1). Así como crecen juntos el grano y la cizaña, conviven en nuestro mundo la praxis de una medicina humana y compasiva con el ejercicio una supra especialización técnica que elimina el valor de la persona. Centrarse exclusivamente en la enfermedad más que en el individuo es un error pues la comprensión de las entidades nosológicas está condicionada por paradigmas y cosmovisiones cambiantes, mientras que el sufrimiento del hombre es una constante.

Muchas han sido las plagas que han azotado esa carne que en palabras de Canetti es "tan fácil de penetrar, desnuda, tierna e indefensa" (2). El objetivo del presente trabajo es describir algunos aspectos históricos y clínicos de una de ellas; el ergotismo, mal de los ardientes o fuego de San Antonio que asoló la Europa medieval en estrecha relación con las hambrunas. Los rostros de este fantasma del pasado, que es un recordatorio de nuestra inmanencia, pueden apreciarse entre otros en el legado pictórico de El Bosco cuyas obras pletóricas de miembros retorcidos por el dolor, monstruos, visiones infernales, pesadillas y sátiras morales que lograron cautivar al muy adusto Felipe II, ejercen aún sobre el espectador contemporáneo un poderoso atractivo similar al vértigo que produce contemplar una sima profunda.

\section{Cornezuelo de centeno y ergotismo}

El ergotismo es el resultado de la toxicidad de los alcaloides del cornezuelo de centeno que son micotoxinas producidas por los géneros Neotyphodium y Claviceps, siendo el principal agente Claviceps purpurea. Este es un hongo externo productor de esporas que parasita más de 600 plantas incluyendo granos económicamente importantes como centeno, trigo, cebada, mijo y avena. Los micelios invaden la semilla y forman excrecencias córneas que miden entre uno y cuatro centímetros, ricas en alcaloides de ergot denominados esclerocios. La intoxicación ocurre cuando éstos son ingeridos por los humanos pues se cosechan junto con el grano sano. La palabra ergot viene del francés antiguo y significa espuela de gallo $(3,4)$. Alrededor de 80 
derivados del ergot provienen de la especie Claviceps, todos tienen un anillo común denominado ergolina. De acuerdo con las modificaciones estructurales se originan tres tipos de sustancias: derivados del ácido lisérgico (ergometrina, ergovasina, metilergonovina), ergopéptidos (ergotamina y bromocriptina) y clavinas (no tienen uso médico) (5). En algunas publicaciones se encuentra mal empleado el epónimo de fuego de San Antonio atribuyéndoselo a la erisipela sin embargo está claro que se refiere al ergotismo (6).

Sus síntomas iniciales son inespecíficos incluyendo debilidad, cefalea, naúsea, vómito y dolor abdominal; posteriormente aparecen las formas gangrenosa o convulsiva. La manifestación más frecuente del ergotismo es la isquemia arterial periférica por vasoespasmo secundario a la estimulación de los receptores periféricos alfa adrenérgicos, dopaminérgicos y serotoninérgicos; a la estimulación directa del músculo liso y a su actividad simpaticolítica central. Clínicamente hay dolor urente intenso, hormigueo, palidez, cianosis, ausencia de pulsos en las extremidades y gangrena seca que conduce a auto amputación, infección o sepsis. La afección es generalmente bilateral y simétrica. El 60 a 70\% ocurre en las extremidades pero puede afectar cualquier vaso $(7,8)$. La ricas descripciones medievales hablan de cuerpos consumidos que dejaban sólo los restos aparentemente carbonizados de las extremidades: "Parecía que las extremidades iban consumiéndose por un fuego interno, se tornaban negras, arrugadas y terminaban por desprenderse" (9). En intoxicación crónica se han descrito además fibrosis retroperitoneal, pulmonar y de válvulas cardiacas así como úlceras anorrectales (7). Así mismo eran muy frecuentes los abortos espontáneos causados por el incremento del tono uterino generado por las toxinas (5). Otra forma típica de presentación es la formicación que consiste en la sensación de insectos que se arrastran debajo de la piel y que puede tener un impacto psíquico aterrador sobre el paciente potenciado por las visibles fasciculaciones musculares asociadas (4). A pesar de que la vida media en plasma de la ergotamina es tan solo de dos horas, produce una vasoconstricción prolongada pues la molécula se une irreversiblemente a los receptores, lo que explica manifestaciones clínicas de isquemia tan prolongadas como 14 días (8).

La segunda forma principal del ergotismo es el convulsivo donde destacan los movimientos anormales que pueden deberse a actividad epiléptica o a contracturas musculares intensas y dolorosas similares a las del tétanos, acompañadas por contracción de los músculos faciales, las cuerdas vocales, el diafragma y por opistótonos. En ocasiones el cuadro evoluciona a estatus convulsivo, coma y muerte. Acompañando a cualquiera de las formas principales de ergotismo pueden presentarse trastornos psiquiátricos como manía, psicosis, visión de colores brillantes, cambios en la percepción del espacio o alucinaciones y que son explicables en vista de la relación estructural de los ergotamínicos con el LSD (10). Una muestra de estas alucinaciones reposa en un fragmento de la Leyenda Dorada de Jacopo da Varagine, arzobispo de
Génova que relata el milagro realizado por los santos Cosme y Damián quienes en sueños de un sacerdote gravemente enfermo trasplantaron en lugar de su pierna gangrenada la extremidad de un moro recién enterrado. Al despertar, el canónigo tenía la pierna sana de un hombre negro (11).

La primera referencia al cornezuelo del centeno proviene de una tabla asiria del año 600 AC donde se le describió como una "pústula nociva en la espiga del grano". Los antiguos griegos reconocían también los efectos tóxicos del moho del centeno, mientras que los romanos no lo consumían al considerarlo un grano inferior como anota Plinio en su Historia Natural (6). El centeno tiene la capacidad de medrar en suelos más pobres y ácidos que los otros granos, por ello sus cultivos se hicieron extensivos en la Edad Media, lo que coincidió con la aparición de las epidemias de ergotismo: en vista de que no es capaz de auto polinizarse sino que se poliniza por el viento, es más susceptible a la acción de las esporas de Claviceps (4). La literatura medieval reporta miles de personas fallecidas, mutiladas o paralizadas. Se cuentan más de 80 epidemias a partir del 945 DC en varios sitios de Europa; ese año fallecieron 20000 personas en la región de Aquitania y en el año 994 perecieron otras 40.000 sólo en la misma región (12). Como es usual los más afectados por la desgracias fueron los pobres pues las clases pudientes se alimentaban fundamentalmente de pan de trigo.

Para la época la naturaleza de la condición era desconocida por lo que se interpretó dentro de un contexto religioso, recibiendo el nombre de "fuego sacro" o ignis sacer. A pesar de las pociones y los amuletos a los que se recurría con frecuencia, la enfermedad seguía avanzando y matando. La única cura conocida parecía ser el peregrinar y recibir cuidados en los monasterios de la Orden Hospitalaria de San Antonio donde los cuidados brindados por los monjes a las víctimas del ergotismo hicieron que los nombres del santo y la enfermedad se vincularan definitivamente.

Como testimonios de estos tiempos sombríos además de las descripciones literarias mencionadas arriba hay abundante material iconográfico sobre el ergotismo que data del otoño de la Edad Media y del Renacimiento. El Bosco dejó numerosas representaciones de miembros gangrenados, hombres mutilados, deformes y las alegorías a alucinaciones con objetos voladores. Es sobrecogedora la contemplación de los dedos crispados en flexión de Cristo en la Crucifixión del retablo de Isenheim (1512-1516) obra del maestro alemán Matthias Grunewald; así como muy elocuentes las extremidades amputadas que aparecen en Los Mendigos de Pieter Bruegel el Viejo (13).

Uno de los temas tópicos en la obra de Hiéronymus Bosch fueron las tentaciones de San Antonio Abad. Aquel fue un monje eremítico egipcio nacido en el 251 DC. A la edad de 20 años vendió todas sus posesiones legándoselas a los pobres y retirándose al desierto a vivir ascéticamente alcanzando la edad de 105 años. Su tumba permaneció desconocida hasta que según la leyenda la localización de los restos fue indicada por un pájaro blanco de pico rojo 
que indicó el sitio exacto y con la ayuda de dos leopardos fue desenterrado en 531 y depositados en Alejandría. En 663 por motivo de la revuelta de los egipcios contra el emperador bizantino Heraclio sus restos fueron llevados a Constantinopla. Durante las cruzadas en 1070 las reliquias fueron llevadas al Delfinado francés a una abadía en Vienne. Sobra señalar la probable naturaleza apócrifa de los restos. En 1090 un noble rico de nombre Gastón prometió ante la tumba del santo usar su fortuna para ayudar a los enfermos de ergotismo si su propio hijo era curado. El milagro se obró y con el dinero del gentilhombre se instituyeron en 1095 un hospital y una cofradía para la cura de enfermos; esta última se constituyó en Orden reconocida formalmente por el Papa Bonifacio VIII en 1297 bajo la regla monástica de San Agustín, ligándola directamente a la autoridad de la Sede Apostólica (14) para despecho de la monarquía capeta que iniciaba sus esfuerzos de centralizar el poder en el trono. Los hermanos antonianos vestían un hábito negro o violeta oscuro con una cruz egipcia en forma de tau sobre la capa y la cogulla; esta cruz recuerda la figura de las muletas sobre las que los sobrevivientes solían continuar su vida; los miembros amputados espontáneamente eran exhibidos como ex votos en las afueras de la abadía (9). Inicialmente la función de la Orden se orientaba al cuidado de las personas con ergotismo pero luego se amplió al cuidado de los niños expósitos, los pobres y los peregrinos. La financiación de la obra se obtenía a partir de las limosnas recogidas de puerta en puerta por los frailes, del recaudo por el culto a las reliquias del cuerpo del santo y de las rentas obtenidas por la venta de ganado porcino marcado con la cruz de San Antonio y cuyos cuidados recaían sobre los feligreses (14). Es sintomático de las debilidades de la naturaleza humana que el culto degenerara en superstición con la proliferación de falsas reliquias con las que los farsantes obtuvieron pingües beneficios a costa de la multitud ignara de campesinos pobres; además pulularon falsos cuestores quienes solicitaban dinero a nombre de la Orden pero cuyo único destino eran sus propias arcas. Más allá de las letanías, la mejoría clínica de los pacientes se debía al cese de la exposición a las toxinas, pues los religiosos brindaban grano limpio a sus dolientes, y al cuidadoso manejo que daban a los muñones de amputación con coberturas que anticiparon a Ambroise Paré. Entre 1095 y el siglo XVI se fundaron cerca de 400 hospitales antonianos desde Suecia hasta Chipre. A pesar de los de la Orden de San Antonio para paliar sus efectos, las epidemias continuaron pues la causa de base aún no se había identificado. El ergotismo convulsivo predominó en áreas al este del Rin mientras que el gangrenoso lo hizo en Francia (10).

En 1596 el médico alemán Wendelin Thelius vinculó el ergotismo al consumo de grano y en 1670 Thuillier relacionó claramente el ergotismo con el cornezuelo de centeno constatando que en aquellos años en los que el cornezuelo era abundante en las cosechas la incidencia del fuego de
San Antonio era mayor; también experimentó con animales a los que administró esclerocios causándoles la muerte. Desgraciadamente Thuillier no publicó sus resultados. Sería Dodar quien aclararía por completo la causa del ergotismo gangrenoso en 1676, mientras que Brunner en 1695 haría lo mismo con el ergotismo convulsivo. Por su parte la Orden de San Antonio fue fusionada con la Orden de los caballeros de Malta en 1771, perdiendo sus últimos monasterios durante la Revolución Francesa y durante la secularización del Sacro Imperio Romano en 1803 (4).

No obstante los avances en la compresión de la enfermedad las epidemias de ergotismo continuaron asolando periódicamente las poblaciones europeas. Sólo comenzaron a disminuir en el siglo XIX: en 1778 tras la muerte de 8000 personas en Sologne, el abate HenryAlexandre Tessier implementara en Francia políticas de salud pública que estimulaban la separación del hongo del centeno, la generalización del consumo de pan blanco de trigo y la sustitución del centeno por la patata americana. Para el siglo XX se reportaron epidemias en los montes Urales en 1926 con 11319 casos de ergotismo, en Irlanda en 1929, en Etiopía en 1977, en la India en 1977 con 140 personas afectadas y una mortalidad de $34 \%$ y nuevamente en Etiopía en 2001 con 18 afectados y tres fallecidos. La última epidemia europea de ergotismo se registró en Francia en 1951 cuando un panadero de Point Saint-Espirit cerca de Avignon, tratando de evadir impuestos compró un cargamento con harina contaminada; el pan elaborado afectó a más de 2000 personas y causó cuatro muertes. Las regulaciones vigentes hoy toleran niveles máximos de ergot entre $0.05 \%$ de peso neto en países como Australia hasta la política de tolerancia cero en el Reino Unido (15). Con lo anterior se ejemplifica el aserto lapidario de Johann Peter Frank en el sentido de que la pobreza es la principal causa de enfermedad: Mientras "el hambre y la enfermedad están pintadas sobre la frente de toda la clase trabajadora... quienquiera que las haya observado no llamará a ninguna de estas personas un hombre libre"; para lograr impactos globales en salud se requiere la voluntad política de los gobernantes.

Hoy el ergotismo no está relacionado con intoxicación alimentaria sino con el uso farmacológico de los derivados del ergot.

La primera descripción del uso médico de los ergotamínicos se hizo en 1582 como estimulante uterino, poco después se descubrió su potencial efecto nocivo sobre la madre acarreando riesgo de muerte por contracción uterina prolongada antes del parto y ruptura uterina Su uso obstétrico en USA data de 1822 cuando John Stearns los implementó para el manejo de hemorragia post parto. A partir de 1868 Woakes la introdujo para manejo de la migraña. A partir de estos beneficios se intensificaron los esfuerzos para la síntesis química del ergot que pudiera permitir una administración clínica no errática a diferencia preparados macerados. En 1906 se sintetizó el primer alcaloide del ergot; la ergotoxina 
y en 1918 se aisló la ergotamina por Arthur Stoll $(4,16)$. Aún hoy la ergotamina se usa en forma extendida para el manejo abortivo de crisis migrañosas y la ergometrina hace parte del arsenal terapéutico para manejo de la hemorragia post parto si bien ha sido desplazada por la oxitocina en razón de su mayor seguridad.

Actualmente se estima una prevalencia de ergotismo del $0.001-0.002 \%$ entre los pacientes que usan ergotamina $(16,17)$. La mayoría de los reportes contemporáneos de ergotismo describen pacientes jóvenes sin factores clásicos de riesgo vascular quienes consumen crónicamente ergotamínicos para migraña y quienes se presentan con isquemia arterial aguda. El cuadro puede estar facilitado por la presencia de sepsis, fiebre, desnutrición, enfermedad coronaria, insuficiencia hepática, tirotoxicosis, hipertensión, embarazo y enfermedad arterial periférica; así mismo el consumo de inhibidores del citocromo CP450 CYP3A4, responsable del $90 \%$ del metabolismo de la ergotamina, puede facilitar el ergotismo. Dentro de estos fármacos se cuentan inhibidores de proteasa como ritonavir, macrólidos, anticonceptivos orales, amiodarona, calcioantagonistas, fluoxetina y ampicilina $(7,17)$. La toxicidad se presentan en cuatro formas: La más frecuente es la intoxicación crónica en paciente que ha ingerido dosis terapéuticas por años; ingestión aguda de dosis altas; ingestión aguda de dosis terapéuticas en pacientes con hipersensibilidad a los fármacos y administración parenteral en pacientes con regulación autonómica anormal. Recientemente se reportaron dos casos de mortalidad materna por uso ergotamínicos: uno por bromocriptina para suprimir la lactancia y otro asociado con utilización de metilergometrina para manejo de hemorragia post parto (5). La forma de ergotismo convulsivo no se ha reportado con el uso de compuestos de ergotamina sintéticos puros.

El diagnóstico diferencial del ergotismo es difícil, requiere alto índice de sospecha basado en la exclusión de diagnósticos diferenciales y en interrogar exhaustivamente por el consumo de fármacos asociados. La arteriografía es el estándar de oro y muestra espasmo arterial usualmente bilateral y simétrico con áreas de estrechamiento abruptas, vasos colaterales en casos de ingestión crónica del ergot y raramente trombos o pseudo aneurismas. Además de suspender los medicamentos culpables no hay manejo estándar. Se recomienda usar aspirina o heparina para prevenir la trombosis; pueden usarse infusiones de nifedipino, prostaglandina $\mathrm{E} 1$, nitroglicerina intra arteriales, $\mathrm{o}$ vasodilatadores orales como IECAS o prazosín. Usualmente el pronóstico con el cuidado actual es bueno pero la isquemia puede tardar en resolver hasta dos semanas (7).

No podemos dejar de mencionar que se ha postulado al ergotismo como el causante de famosos casos de brujería. En el año 1600 la supuesta bruja inglesa Alice Trevisard tenía según las descripciones de la época los dedos de las manos y los pies gangrenados como correspondía al ergotismo gangrenoso. Durante los juicios de brujas de
Finmark en Noruega 137 personas fueron juzgadas y dos terceras partes ejecutadas; se dispone de registros donde 42 en los casos estaba implicado el consumo de leche con cuerpos semejantes a granos de color negro que pudieron haber sido esclerocios (10). A finales de diciembre de 1691 en Salem 8 muchachas presentaron lenguaje descoordinado, movimientos convulsivos, posturas extrañas, cambios de pigmentación en la piel (la marca del diablo), ceguera transitoria, sensación de urente y visiones como de bolas de fuego o de multitudes con túnicas blancas brillantes. Ello conllevó a los famosos juicios donde murieron 20 personas; 19 fueron colgadas y un anciano fue aplastado bajo piedras. La hipótesis de la intoxicación por cornezuelo de centeno es plausible en vista de que había en ese entonces centeno salvaje a lo largo de la costa atlántica de Estados Unidos, de la coexistencia de compromiso cutáneo y neurológico; sin embargo no es plenamente satisfactoria en vista de que por la epidemiología se esperaría un mayor número de personas afectadas. La realidad no se conoce con certeza y toda discusión es meramente especulativa $(18,19)$.

$\mathrm{Al}$ ergotismo se le ha adjudicado además la responsabilidad de los estados extáticos de los iniciados durante los misterios de Eleusis (19) y se ha señalado como posible culpable de las epidemias registradas en los siglos XIV y XVII de manía de la danza, donde cientos de personas sufrieron una compulsión agónica por bailar, llegando a hacerlo por horas o días, incluso hasta morir (20). A este respecto conviene guardar prudentes reservas. Como nos advierte la historiadora norteamericana Mary Lindeman "sin duda es loable el intento de historiar la enfermedad, pero uno se pregunta si son ésas las conclusiones totalmente correctas. Sería bastante simplista aplicar explicaciones "científicas" modernas a fenómenos culturales complejos" (21).

Para concluir quisiéramos mencionar que mientras el emperador Federico Barbarroja durante el asedio de Tortona de 1155 optó por contaminar con cadáveres humanos la única fuente de agua de la ciudad sitiada y mientras los mongoles catapultaban cuerpos de víctimas de la peste sobre Caffa en 1346, ya los asirios en el siglo VI antes de Cristo habían descubierto la guerra biológica usando ergotamínicos para envenenar los pozos de sus enemigos (22).

\section{Corolario}

En palabras de Octavio Paz: "la historia es conocimiento que se sitúa entre la ciencia propiamente dicha y la poesía. El saber histórico no es cuantitativo ni el historiador puede descubrir leyes históricas. La historia nos da una comprensión del pasado y a veces del presente. Más que un saber es una sabiduría" (23).

El devenir histórico del mal de los ardientes nos revela que a pesar del cambio de rostro de las entidades nosológicas y de las cosmovisiones, el hombre es un ser cuya existencia está condicionada por una carne frágil que palpita; el encuentro dialogante con esta realidad en el otro que sufre es el fundamento insustituible del quehacer médico. 


\section{Agradecimientos}

A mis padres, mi esposa y mi hija por su amor incondicional; a toda prueba.

\section{Referencias}

1. Montaigne M. De la presunción. En: Ensayos completos. Montojo A, traductora. Madrid: Cátedra; 2013. p. 630-657.

2. Canetti E. Masa y poder. Del Solar JJ, traductor. Barcelona: Debolsillo; 2011.p. 69-70

3. Illana-Esteban C. El cornezuelo del centeno (I): Biología, historia y ergotismo. Bol. Soc. Micol. Madrid 2008; 32: 293-306

4. Quesada Díaz A, Ortega Díaz A. El cornezuelo del centeno a lo largo de la historia: mitos y realidades. Pasaj. Cienc. 2011; 14: 16-25

5. Velásquez-Penagos JA, Gómez-Jimenez JM,Agudelo-Jaramillo B. Ergotismo del sistema nervioso central. Reporte de dos muertes maternas asociadas a vasoespasmo cerebral por medicamentos derivados del ergot en Antioquia, Colombia y

revisión de la literatura. Rev Colomb Obstet Ginecol 2016; 67: 231-241

6. Carlton MW, Kunkel DB. St. Anthony's Fire: Eponym misused. Acad Emerg Med 1995; 2: 1114-1115

7. Vallejo FJ, Gómez JF, Tamayo N. Isquemia aguda de miembros inferiores secundaria a ergotismo. Rev Colomb Cardiol 2011; 18: 350-352

8. Zavaleta EG, Fernández BB, Grove MK, Kaye MD. St. Anthony's Fire (Ergotamina Induced Leg Ischemia) A case eport and review of the literature. Angiology 2001; 52 (5): 349-356

9. Laval E. Sobre las epidemias del fuego de San Antonio. Rev Chil Infect 2004; 21 (1): $74-76$

10. Torbjorn A, Elvevag B. Ergotism in Norway. Part 1: The symptoms and their interpretation from the late Iron Age to the seventeenth century. Hist Psych 2013; 24: $15-33$
11. Lee MR. The history of ergot of rye (Claviceps purpurea) I: From antiquity to 1900. J R Coll Physicians Edinb 2009; 39: 179-84

12. De Costa C. St. Anthony's fire and living ligatures: a short history of ergometrine Lancet 2002; 359: 1768-1770

13. Battin J. Le fue Saint-Antoine ou ergotisme gangreneux et son iconographie medievale. Histoire des Sciences Medicales. 2010; 44 (4): 373-382.

14. Argente C. La Orden Hospitalaria de San Antón en la diócesis Baeza-Jaén. Cuad Est Mediev. 1974 (2): 37-57.

15. Belser-Ehrlich S, Harper A, Hussey J, Hallock R. Human and cattle ergotism since 1900: Symptoms, outbreaks and regulations. Toxicol Ind Health 2013; 29: 307-316

16. Van Dongen PWJ, De Groot A. History of ergot alkaloids from ergotism to ergometrine. Eur J Obstet Gynecol Reprod Biol 1995; 60: 109-116

17. Ruano-Calderón LA, Zermeño-Pohls F. Ergotismo. Presentación de un caso y revisión de la bibliografía. Rev Neurol 2005; 40: 412-416

18. Mundra LS, Maranda EL, Cortizo J, Augustynowicz A, Shareef S, Jimenez J. The Salem Witch Trials- Bewitchment or Ergotism. JAMA Dermatology 2016; 152 (5): 540

19. Illana-Esteban. El cornezuelo del centeno (II):Brujería, medicina y contenido en alcaloides. Bol. Soc. Madrid 2009; 33: 263-272

20. Waller J. The art of medicine. A forgotten plague: making sense of dancing mania. Lancet 2009; 373: 624-625

21. Lindemann M. Medicina y sociedad en la Europa moderna 1500-1800. Pérez A, traductora. Madrid: Siglo XXI Editores; 2001.p. 1-68.

22. Klassen-Fischer MK. Fungi as bioweapons. Clin Lab Med 2006: 26: 387-395

23. Paz O. El ogro filantrópico: historia y política 1971-1978. México: Joaquin Mortiz; 1979. p. 20-21. 